\title{
Factibilidad de la utilización de recursos tecnológicos en la implementación del eje transversal cultura ambiental para el desarrollo sostenible en la Educación General Básica de Costa Rica
}

\author{
Feasibility in Using Technological Resources for Implementing the Environmental \\ Culture Cross-Cutting Factor for Sustainable Development in the \\ Costa Rican Basic General Education
}

\author{
Lorna Matarrita Román ${ }^{1}$ \\ Bri bri School \\ Alajuela, Costa Rica \\ lor1102@hotmail.com \\ Jessica Serrano Calvo ${ }^{2}$ \\ Sorek Centro Educativo \\ San José, Costa Rica \\ yk0387@hotmail.com \\ María Isabel Torres Salas ${ }^{3}$ \\ División de Educología \\ Centro de Investigación en Educación \\ Universidad Nacional \\ Heredia, Costa Rica \\ isabeltorresr@yahoo.com \\ Giselle Léon León ${ }^{4}$ \\ División de Educología \\ Centro de Investigación en Educación \\ Universidad Nacidonal \\ Heredia, Costa Rica \\ leongiselle@hotmail.com
}

Recibido 19 de junio de 2012 • Corregido 16 de setiembre de 2012 • Aceptado 07 de noviembre de 2012

Licenciada en enseñanza de las Ciencias, Universidad Nacional de Costa Rica, docente de secundaria, Bri bri School.

2 Licenciada en enseñanza de las Ciencias, Universidad Nacional de Costa Rica, docente de secundaria, Sorek Centro Educativo.

3 Académica de la División de Educología, Universidad Nacional de Costa Rica. Bachiller en Ciencias de la Educación, bachiller en la Enseñanza de la Química, licenciada Enseñanza de la Química y máster en Psicopedagogía.

4 Académica de la División de Educología, Universidad Nacional de Costa Rica. Máster en Administración Educativa, bachiller en la Enseñanza de las Ciencias y Licenciada en Ciencias de la Educación con Énfasis en Didáctica de Ciencias. Estudiante del Doctorado en Educación de la Universidad Autónoma de Durango, México. 
URL: http://www.una.ac.cr/educare

Resumen. El propósito de esta investigación fue analizar si, en la implementación del eje transversal cultural ambiental para el desarrollo sostenible, que enfatiza en problemáticas ambientales relacionadas con los contenidos del programa de Ciencias de séptimo ${ }^{5}$ año de la Educación Básica, puede ser factible la utilización de recursos tecnológicos. El diseño de la investigación fue cualitativo con enfoque dominante, con algunos elementos de corte cuantitativo, específicamente en el diseño de instrumentos y algunas técnicas de análisis de datos. El tipo de estudio se desarrolló con un enfoque multimétodo, tendencia que ha ido perfilando un estilo de investigación en el que se integran distintos métodos en un mismo diseño. Para ello, se determinaron cuáles fueron las estrategias didácticas y su relación con la tecnología y el eje cultura ambiental para el desarrollo sostenible, utilizadas por 6 docentes de Ciencia que se desempeñaron en séptimo año en instituciones públicas, localizadas en la provincia ${ }^{6}$ de Heredia del Valle Central de Costa Rica, así como la opinión de 20 estudiantes de ese mismo nivel. Dentro de los principales resultados se indican las opiniones de estudiantado, que expone su gran interés por recibir las lecciones que incorporen recursos tecnológicos. Por otro lado, algunos docentes se muestran no muy favorables ante el tema o desinteresados al respecto, además de no estar capacitados en su uso. Asimismo se identificó que este personal docente encuestado no desarrolla el eje curricular.

Palabras claves. Estrategias didácticas, TIC, recursos tecnológicos, cultura ambiental, desarrollo sostenible.

Abstract. The purpose of this research was to analyze whether the use of technological resources may be feasible in the implementation of the environmental culture cross-cutting factor for sustainable development, which focuses on environmental issues related to the contents of the Science study program for the seventh year of the basic general education. The research design is qualitative with a dominant approach and uses some quantitative elements specifically in the design of instruments and some data analysis techniques. The type of study was developed with a multi-method approach; a trend that has been shaping a research style which integrates various methods in a single design. For this, we identified the didactic strategies and their relationship to both, technology and the environmental axis for sustainable development, used by six Science teachers of the $7^{\text {th }}$ grade, in public institutions of the province of Heredia, Central Valley, Costa Rica, as well as the opinion of 20 students from that same grade. The main results include the opinions of the students, who showed a considerable interest in classes where technological resources are used. However, teachers do not show great interest or positive opinions on this matter; in addition, they are not well trained on the use of technological resources. It was also identified that the teaching personal who participated in the study do not develop this curricular axis.

Keywords. Teaching strategies, TIC, technological resources, environmental culture, sustainable development.

\footnotetext{
Primer nivel de secundaria costarricense.

6 Organización territorial de Costa Rica, distribuida en 7 provincias.
} 


\section{Introducción}

A nivel mundial, el ser humano ha puesto en peligro su propia vida a causa del modo en que desarrolla sus relaciones con el ambiente. Algunas consecuencias de las problemáticas que genera el desarrollo pueden ser el deterioro de la capa de ozono, el efecto invernadero y el cambio climático. Esto es importante de rescatar, ya que, en la mayoría de los casos, son imperceptibles para la población y se hace necesario una participación más activa y determinante ante esos problemas (Martínez, 2010).

Según Borderías y Martín (2006) el término "ambiente" involucra aspectos que influyen de manera directa con el desarrollo de la vida humana, animal y vegetal. Dada la importancia que este conlleva como bien común, su constante deterioro amenaza el bienestar de todos los seres vivos. La problemática ambiental es un tema que concierne a todo ser humano por igual, debido a que de una forma u otra, todas las personas participan en la destrucción de los sistemas naturales de vida, de igual manera se debe trabajar en conjunto (incluidos todos los sectores de la población) para la búsqueda de soluciones.

La educación, es uno de estos sectores importantes, siendo una alternativa en el manejo de los problemas de cualquier índole. El deterioro ambiental que está sufriendo el planeta no es la excepción, por ello es urgente educar a la población acerca del peligro que representa continuar con un estilo de vida poco amigable con la naturaleza: las formas de consumo y producción ha provocado daños a la atmósfera, los ríos, los cultivos. En fin, se atenta contra las diferentes formas de vida que hay en la tierra. Según Boff (2007), a medida que el planeta se va calentando, aumentan los huracanes, el nivel del mar, se extinguen muchas especies y se pronostica que se incrementarán las guerras por la posesión de los recursos.

A pesar de la realidad descrita, la educación sigue siendo la mejor opción con que cuenta la humanidad para propiciar cambios: en ese caso, lo ideal es que, como parte del currículo, se sensibilice al educando en temas ambientales.

Partiendo del hecho de que el Ministerio de Educación Pública de Costa Rica propone, entre sus ejes transversales, uno relacionado con la "Cultura Ambiental para el Desarrollo Sostenible" (MEP, 2005, p. 2), el personal docente tiene el deber de desarrollar los contenidos relacionándolos con este eje.

Para ello es necesario utilizar diferentes estrategias que incentiven al estudiantado a tener interés y así lograr un aprendizaje más significativo y vivencial en las aulas. Uno de esos recursos puede ser la implementación de la tecnología, pues dentro de muchos beneficios ha permitido implementar cambios en los métodos de enseñanza e, incluso, en la forma de concebir el aprendizaje, donde cada vez más es el propio estudiante quien toma el control del proceso, los materiales y los recursos, adaptándolos a sus obligaciones, necesidades y posibilidades (González, 2005). 
URL: http://www.una.ac.cr/educare

Por otra parte, los educadores se enfrentan a un mundo de innovaciones, al desarrollo de su práctica docente a través de la integración de las tecnologías como un recurso más en el proceso de enseñanza y aprendizaje. Estas les permiten promover y facilitar la actitud participativa y creadora de los educandos, ocasionando en ellos una verdadera transformación en el proceso al cederles el papel protagónico.

Tomando en consideración lo antes expuesto, se sabe que en la actualidad los recursos tecnológicos y redes sociales son de gran atracción para las personas adolescentes y las adolescentes, razón por demás interesante para tratar de plantear estrategias que utilicen estos recursos, influir positivamente en los procesos de enseñanza y aprendizaje del estudiantado y en los procesos de planeamiento y desarrollo de clases del personal docente. Lo expuesto en líneas anteriores motiva la siguiente pregunta de investigación:

¿Cómo realizar un análisis sobre la factibilidad de utilizar recursos tecnológicos en la implementación del eje transversal cultura ambiental para el desarrollo sostenible en algunos contenidos del programa de Ciencias, para facilitar la educación ambiental?

\section{Objetivo general}

Analizar si el uso de recursos tecnológicos es factible en la implementación del eje transversal cultura ambiental para el desarrollo sostenible en los contenidos del programa de Ciencias, con estudiantes de séptimo año de un colegio académico de la provincia de Heredia, con el fin de facilitar el acceso a la educación ambiental de una manera innovadora.

\section{Objetivos específicos}

1. Identificar las estrategias que utilizan docentes de Ciencias de séptimo año, para enseñar los contenidos: uso racional de la energía, la atmósfera, fenómeno del Niño y de la Niña, la capa de ozono, hidrósfera y deterioro del planeta tierra, que están relacionados con el eje curricular cultura ambiental para el desarrollo sostenible.

2. Describir las estrategias metodológicas que utiliza la docente de la sección en estudio para desarrollar las clases de Ciencias.

3. Determinar el interés que tiene el estudiantado de una sección de séptimo en cuanto a la utilización de recursos tecnológicos.

\section{Referentes conceptuales}

Durante los años 1940, el aumento de la población y el inicio del proceso de ampliación de la frontera agrícola reducen significativamente los espacios donde se localizaban naturalmente 
la flora y la fauna. De ahí que, con la intención de que la biodiversidad continúe su desarrollo, se hace necesaria la creación de espacios naturales protegidos. Durante estos periodos surge una legislación para mantener estos recursos; pero, dado que la tarea no es fácil, para los años setenta se inicia el proceso de establecimiento y consolidación del Servicio de Parques Nacionales, el Departamento de Vida Silvestre y el de la Dirección Forestal. En esa misma época, el Estado considera necesaria la creación del Sistema Nacional de Áreas de Conservación (Martínez, 2010).

En los últimos años, Costa Rica se ha destacado, internacionalmente, por ser uno de los países que desarrolla mayor conciencia en preservación de los recursos naturales y por la creación de espacios naturales, protegidos por una legislación.

\section{Educación ambiental}

Se puede definir la educación ambiental como un proceso que suscita la concienciación de la población; comprende el desarrollo político, social, físico, cultural, espiritual y natural como una unidad y la utilización, de manera racional y sostenible, de los recursos, de manera que se garantice la supervivencia de la humanidad consigo misma y con la naturaleza (Salgado, 2009).

En esta misma línea, Machado (2002) dice que “(...) la educación ambiental es, sin duda, uno de los medios más indicados para el rescate de valores que incluyen el respeto por la diversidad cultural y biológica, fundamental para la conservación y para la convivencia armónica de las diferentes culturas con la naturaleza (...)" (p. 51). Es decir, la educación es el medio por el cual se pueden cambiar las prácticas cotidianas, el estilo de vida, las formas de consumo y producción; sin embargo, debido a que es difícil cambiar las formas de comportamiento de las personas en la sociedad, es urgente iniciar cuanto antes programas educativos.

Para lograr lo anterior, hay que introducir la educación ambiental en todos los niveles formales y no formales, ya que esta tiene como propósito relacionar al ser humano con su medio ambiente, con su realidad y propiciar un cambio de actitud, una toma de conciencia de la importancia de conservar el planeta para el futuro y para mejorar la calidad de vida actual.

\section{Ejes transversales}

Los ejes transversales dentro del marco de la educación costarricense deben favorecer el desarrollo de valores, actitudes, habilidades y destrezas cognoscitivas de las personas; debido a los cambios científicos, tecnológicos, ambientales, económicos y sociales del mundo, es decir, debe tomar como gran eje transversal los valores fundamentales del humanismo (MEP, 2005). 
URL: http://www.una.ac.cr/educare

La transversalidad es una fuente de riqueza globalizada que parte de dimensiones ambientales (sociocultural, política, religión, entre otros). La diversificación fusiona o alianza las asignaturas enseñadas (Español, Arte, Música, Biología, Física, Química, Cívica, entre otras) en el aula; esta es la característica interdisciplinaria de la estructuración racional de la transversalidad en el proceso enseñanza y aprendizaje, así como la inclusión y vivencia de los valores.

Por medio de la transversalidad, se aprovechan las oportunidades que ofrece el currículo, para adecuarlas a las necesidades de las poblaciones, de manera que no se saturen los programas de estudio y se logren los objetivos planteados (MEP, 2005).

Dada la importancia de formar individuos no solo en las áreas académicas, sino también formarlos en cuanto a las problemáticas en que se ven inmersos, el Ministerio de Educación Pública (MEP) define los siguientes temas transversales: "Cultura Ambiental para el Desarrollo Sostenible, Educación Integral de la Sexualidad, Educación para la Salud y Vivencia de los Derechos Humanos para la Democracia y la Paz" (MEP, 2005, p. 2).

Por la temática del estudio se usó el eje cultura ambiental para el desarrollo sostenible, el cual indica:

Cultura ambiental para el desarrollo sostenible

La educación ambiental se considera como el instrumento idóneo para la construcción de una cultura ambiental de las personas y las sociedades, en función de alcanzar un desarrollo humano sostenible, mediante un proceso que les permita comprender su interdependencia con el entorno, a partir del conocimiento crítico y reflexivo de la realidad inmediata, tanto biofísica como social, económica, política y cultural.

Tiene como objetivo que, a partir de ese conocimiento y mediante actividades de valoración y respeto, las y los [sic] estudiantes se apropien de la realidad, de manera que, la comunidad educativa participe activamente en la detección y solución de problemas, en el ámbito local, pero con visión planetaria. (MEP, 2005, p. 3)

La realidad ambiental nacional y mundial está sufriendo a causa de la negligencia humana; afecta tanto la sociedad, la economía, la política y la cultura que enriquece nuestro patrimonio. Por esta razón, se debe reflexionar al respecto, para actuar pronto y con disposición de manera que se pueda concienciar sobre sus consecuencias. 


\section{Estrategias didácticas para la enseñanza de las Ciencias}

Las estrategias de enseñanza son procedimientos (conjunto de pasos, operaciones o habilidades) que el sujeto aprendiz emplea en forma consciente, controlada e intencional, como instrumento flexible para significativamente solucionar problemas (Díaz Barriga, Castañeda y Lule, 1986; Gaskins y Elliot, 1998, citados por Díaz y Hernández, 2002). Actualmente se conocen diferentes tipos de estrategias didácticas que pueden ser modificadas y ajustadas de acuerdo con la creatividad y pensamiento del docente, en la perspectiva de ayudar al estudiante a comprender los procesos de enseñanza y aprendizaje mediante diversas actividades tales como la realización de mapas conceptuales, una presentación animada, juegos interactivos, entre otros. Favorecen al estudiantado en la comprensión de la materia y al sujeto docente, no solamente en la satisfacción de alcanzar lo esperado, sino también en la evidencia de los logros.

\section{La tecnología educativa como recurso didáctico}

Se sabe que los computadores y las redes son una oportunidad para que los profesores, las profesoras y educandos puedan participar activamente de las transformaciones que vive la sociedad y se ha dejado de lado el pensamiento de que la informática es un recurso a cargo de los que saben de eso. Galvis (2004) indica al respecto:

Hay educadores que siguen aferrados a esquemas que se centran en transmitir y asimilar conocimiento comprobado, con lo que para ellos las TIC son una buena ocasión para poner a disposición de más gente -y con recursos multimedia- aquello que creen que deben enseñar. Y su gran reto es hallar buenas herramientas para difundir mejor lo que saben o lo que desean que aprendan sus alumnos. También tienen el reto de hallar los mejores medios para hacer llegar a los destinatarios lo que han mediatizado, dependiendo de su distribución geográfica y del acceso que tengan a recursos informáticos. (Los educadores y la informática, párr. 1)

De acuerdo con lo antes planteado, la tecnología educativa se postularía como uno de los campos en los cuales el educador puede hacer uso de esta como recurso didáctico para el desarrollo de procesos activos en la enseñanza y el aprendizaje. Al respecto, la mayor preocupación la constituye el papel que debe cumplir el personal docente en la tecnología educativa

Olier (s. f.) explica que el uso de las tecnologías en un salón de clases no sirve de nada si quien ejerce la docencia no le da el uso pedagógico que se merece, lo cual depende de su mediación pedagógica, específicamente de utilizarla, de manera complementaria, como estrategia didáctica que permita que los educandos sean participes del proceso y no solo receptores. 
URL: http://www.una.ac.cr/educare

El alumno se vuelve gestor de su propio proceso de aprendizaje; el profesor se convierte en facilitador, colaborador y orientador de ese proceso. En nuestra opinión esta esperanza es la más probable y la más interesante de todas; un cambio de esta naturaleza en el ambiente de aprendizaje es estructural, afecta notablemente el clima escolar y posibilita formas de trabajo que probablemente favorecen la construcción de conocimiento y la práctica de habilidades y destrezas deseables. (Romero, 2009, p. 4)

Es decir, se pretende pasar de un proceso que centrado en la actividad del docente a uno que gire alrededor del educando, sin pensar que el rol del docente deba desaparecer por completo, sino que servirá de apoyo para crear ambientes educativos agradables y ricos en oportunidades para llegar al conocimiento a partir de la experiencia.

Con el fin de garantizar un entorno satisfactorio del aprendizaje de los estudiantes y las estudiantes a través de la tecnología educativa, el personal docente podría usar un sistema de trabajo en el cual el educando sea capaz de llegar a construir su propio conocimiento a partir de la guía del docente, dejando de antemano la idea de ser participes de una tendencia algorítmica en la tecnología educativa.

En contraparte a la tendencia algorítmica, se podría pretender que el estudiante y la estudiante, a partir del proceso de enseñanza y aprendizaje, sea participe de la construcción de su propio conocimiento, utilizando estrategias y materiales propios de la tecnología educativa; ese aprendizaje puede lograrse desde una perspectiva mixta, donde el docente interactúa con su alumnado y él mismo, a su vez, con un grupo de estudiantes, los cuales trabajan activamente.

\section{Las tecnologías de la información y la comunicación (TIC) en el proceso de enseñanza y aprendizaje}

En la actualidad, los sistemas educativos de todo el mundo tienen el reto de utilizar las TIC para ofrecer a sus alumnos y alumnas las herramientas y conocimientos necesarios que se requieren en el siglo XXI. Al respecto, Bautista (2007, citando a la UNESCO, 2004) menciona:

(...) En el área educativa, los objetivos estratégicos apuntan a mejorar la calidad de la educación por medio de la diversificación de contenidos y métodos, promover la experimentación, la innovación, la difusión y el uso compartido de información y de buenas prácticas, la formación de comunidades de aprendizaje y estimular un diálogo fluido sobre las políticas a seguir. Con la llegada de las tecnologías, el énfasis de la profesión docente está cambiando desde un enfoque centrado en el profesor que se basa en prácticas alrededor del pizarrón y el discurso, basado en clases magistrales, hacia una formación centrada principalmente en el alumno dentro de un entorno interactivo de aprendizaje. (párr. 1 y 2) 
De igual manera opinan Palomo, Ruiz y Sánchez (2006), quienes indican que las TIC ofrecen la posibilidad de interacción que pasa de una actitud pasiva por parte del alumnado a una actividad constante, a una búsqueda y replanteamiento de contenidos y procedimientos.

Asimismo, destacan que el uso de las TIC favorece el trabajo colaborativo entre iguales, no solamente por el hecho de tener que compartir una computadora con un compañero o compañera, sino por la interacción en la construcción del conocimiento y su socialización.

- (...) la experiencia (...) demuestra día a día que los medios informáticos de que se dispone en las aulas favorecen actitudes como ayudar a los compañeros, intercambiar información relevante encontrada en Internet, resolver problemas a los que los tienen (...). Estimula a los componentes de los grupos a intercambiar ideas, a discutir y decidir en común, a razonar el por qué de tal opinión (...). (Palomo et al., 2006, p. 18)

\section{Metodología}

La investigación se realizó en el 2011 en un grupo de séptimo año de la Educación General Básica, conformado por 20 estudiantes, de una institución pública con jornada diurna de la provincia de Heredia. Además se consideró la docente de Ciencias del grupo y cinco docentes más de otras instituciones que impartieron ese nivel; la muestra es reducida porque se pretendió un estudio a profundidad y no la generalización de los resultados.

El trabajo se desarrolló con un enfoque multimétodo: “Esta tendencia ha ido perfilando un estilo de investigación en el que se integran distintos métodos en un mismo diseño (...)" (Pérez, 2007, p. 11). En este caso prevaleció la investigación cualitativa, con la utilización de ciertos elementos del enfoque cuantitativo.

La información recolectada en el proceso de investigación se obtuvo mediante diferentes instrumentos (entrevista semi estructurada, registro anecdótico y cuestionario). Se aplicó un cuestionario a 5 docentes de Ciencias de instituciones públicas y diurnas, con el fin de identificar las estrategias metodológicas y los recursos tecnológicos que empleaban en el desarrollo de sus clases, así como conocer si incluían el eje transversal cultura ambiental para el desarrollo sostenible.

Además, el registro anecdótico se utilizó para realizar las observaciones, que se llevaron a cabo durante el desarrollo de las lecciones de Ciencias en dos momentos diferentes, con el fin de determinar cuáles estrategias metodológicas y qué recursos didácticos utilizaba la docente de Ciencias del grupo en estudio, en el salón de clases y si implementaba el eje transversal en cuestión.

De igual manera se observó la forma en que se desenvuelve el estudiantado durante las lecciones, elemento que se contrastó con la aplicación de un cuestionario a estudiantes de séptimo año. 
URL: http://www.una.ac.cr/educare

Por otra parte, con el fin de conocer su interés por utilizar recursos tecnológicos para desarrollar las lecciones de Ciencias, incluyendo el eje transversal cultura ambiental para el desarrollo sostenible, a la docente de Ciencias del grupo en estudio se le aplicó una entrevista semi estructurada y, por último, a los educandos se les aplicó un cuestionario para determinar su interés por la utilización de recursos tecnológicos.

En el análisis de resultados se utilizó la triangulación descriptiva como medio de interpretación de datos, lo cual permitió contrastar la información. También se analizaron los datos de las entrevistas y cuestionarios, para luego interpretar los datos y llegar a conclusiones. Todos los instrumentos fueron diseñados por las investigadoras y validados mediante pruebas piloto.

\section{Resultados y discusión}

De acuerdo con los resultados del cuestionario aplicado a cinco docentes de Ciencias de otras instituciones, con respecto a la percepción que tenían acerca de las estrategias didácticas que utilizaban en sus clases, se evidenció que se confunde estrategia didáctica recursos. También se encontró que las estrategias utilizadas fueron repetitivas, aspecto preocupante porque como afirman Delgado y Solano (2009), las estrategias de enseñanza son todas las ayudas planteadas por el personal docente, con el objetivo de facilitar un procesamiento más profundo de la información y, por ende, mayor aprendizaje. A esos efectos, si se desarrollan clases donde no media una variada gama de estrategias didácticas, probablemente no se está dando la oportunidad a que el estudiante logre un buen aprendizaje.

Con respecto al uso de recursos se encontró que la mayoría de docentes de Ciencias utiliza, con mucha frecuencia, el proyector de multimedia, la computadora, acceso a Internet, películas, entre otros de índole tecnológica. Manifestaron que estos son un medio que facilita su labor docente, ya que ayuda al estudiantado a tener mayor comprensión de conceptos abstractos difíciles de entender, pero que con la ayuda de imágenes, videos, cuentos y películas, se experimenta un aprendizaje más vivencial. Esta es una de las razones por la que es muy importante la implementación de estas tecnologías en el sector educativo; en contraste, se debe indicar que los(as) docentes encuestados(as) indican no vivenciar el eje transversal cultura ambiental para el desarrollo sostenible.

Por otra parte de acuerdo con los resultados obtenidos de las observaciones realizadas en el desarrollo de las lecciones, se encontró que las estrategias utilizadas por la docente de Ciencias del grupo de séptimo año, se basaban en dictados, mediados por explicaciones, ejemplos y resúmenes. Por otra parte, sus estudiantes no participaban la mayoría del tiempo, solo en algunos momentos; se mostraban desatentos, conversando de otros temas, enviando mensajes por celular -no parecían estar identificados con la clase-, y no tenían roles muy claros de lo que debían hacer. 
Se puede decir que las estrategias manejadas por la docente y las conductas observadas en los estudiantes durante el desarrollo de la clase están asociadas a modelos academicistas, en donde el énfasis está en el contenido y no en un proceso que le permita al estudiante construir su conocimiento; además, este tipo de estrategias no facilita que el estudiantado relacione los contenidos vistos en clase con la realidad, para que pueda ver su aplicación y de esta forma cobre significado su aprendizaje.

En contraste, aunque la institución cuenta con varios recursos (videoproyector, TV, laboratorio de cómputo, laboratorio de Ciencias), la docente de Ciencias utilizó únicamente como material de apoyo la pizarra, las fotocopias y el libro de texto.

En relación con la entrevista hecha a la docente de Ciencias respecto a la utilización de recursos tecnológicos para implementar el eje transversal en estudio, se evidenció la falta de interés que presentó en cuanto a la utilización de los recursos tecnológicos. Manifestó que estos recursos son una herramienta como cualquier otra que puede ser utilizada a la hora de desarrollar las clases; además, no cree que haya beneficio alguno en el proceso de enseñanza y aprendizaje, al implementar el eje transversal cultura ambiental para el desarrollo sostenible, mediante el uso de recursos tecnológicos. Por ende indica no implementar el eje, a pesar de que algunos de los temas del nivel lo permiten.

Entre las limitantes que el profesorado de Ciencias expresa que tiene, en lo que respecta al uso de recursos tecnológicos están: el tiempo y la forma tradicional de evaluación que impone el sistema educativo y la falta de capacitación respecto al uso de estos recursos. Justifica el uso de fotocopias para impartir la materia, porque afirma que agiliza y porque resulta muy cansado, para el profesorado, escribir en la pizarra o dictar y, para los estudiantes y las estudiantes, copiar en el cuaderno. Sin embargo, la docente reconoce que los recursos tecnológicos -como las computadoras, el internet, los juegos electrónicos, los videos, el proyector de multimedia, entre otros- son atractivos para el alumnado a la hora de recibir lecciones. Indica que en sus clases no utiliza ninguno de estos, por las limitaciones que mencionó anteriormente.

Finalmente, la docente propone que para implementar el eje transversal Cultura ambiental para el desarrollo sostenible es necesario desarrollar actividades en las cuales el estudiantado participe de forma activa en su propio proceso de enseñanza y aprendizaje, donde sea él mismo quien deba construir metodologías afines con los modelos constructivistas. En contraste, lo propuesto es distinto a lo que se observó en el desarrollo de sus clases y sus propias respuestas.

Además, en el desarrollo de la entrevista se comprobó que hay desconocimiento en cuanto al uso de las tecnologías educativas, razón por la que probablemente alberga prejuicios hacia el uso de estas herramientas.

De igual manera, como resultado del cuestionario aplicado a los estudiantes y las estudiantes de séptimo año, en la pregunta relacionada con las estrategias y los recursos que utilizan docentes de Ciencias en sus clases, se encontró que 13 de los 16 estudiantes afirman que el profesorado nunca utiliza canciones, lecturas, películas y grabaciones en el desarrollo 
de la clase. Por otra parte, en cuanto al uso de cuestionarios, libros de texto y explicaciones, la mitad coincide en que siempre son utilizados en las lecciones de Ciencias; la otra mitad expresa que la mayor parte del tiempo no se hace uso de estos recursos. Además, al triangular la información, se encontró que la mayoría de estudiantes coincide en que el recurso que utiliza con más frecuencia la docente es la pizarra y las fotocopias y, en menor cantidad, los otros recursos como (grabaciones, power point, imágenes y libros de texto). También se pudo constatar que los recursos como películas, juegos tecnológicos, redes sociales y cuentos no se utilizan para el desarrollo de sus lecciones.

Lo anterior podría estar afectando el rendimiento académico, el cual es bastante bajo, según lo comentado por la docente en la entrevista. Además se puede afirmar que con las estrategias y recursos didácticos que utiliza para desarrollar las lecciones, lo más probable es que favorezca solamente a algunos estilos de aprendizaje de sus estudiantes.

Por otra parte se puede deducir que la forma en que la docente imparte sus lecciones responde a un modelo de enseñanza y aprendizaje centrado en el contenido y el producto, y no en el estudiante y el proceso. En estas lecciones no se promueve una concepción constructivista, en la cual, según Chadwick (2001), se vive un proceso activo de parte del educando, por medio del que se hacen conexiones, amplían, restauran e interpretan y, por lo tanto, construyen el conocimiento desde los recursos de la experiencia y la información que recibe.

A pesar de lo indicado antes, la totalidad del grupo de estudiantes en estudio expresó que tiene interés por recibir las lecciones de Ciencias con recursos tecnológicos, lo que demuestra que la utilización de la tecnología en las experiencias de enseñanza en general, es un factor que podría despertar la motivación, aspecto esencial para lograr el aprendizaje en cualquier tema. De ahí que desde un video hasta una red social son recursos que se pueden adecuar al proceso de enseñanza y aprendizaje, y así motivar al estudiantado a mantenerse dentro de las aulas, no solo recibiendo contenidos "trasmitidos" por el personal docente, sino apropiándose de estos por medio de estrategias y recursos que despierten su interés, de manera que la comprensión de los mismos les permita aplicarlos en la solución de problemas de la vida diaria.

La búsqueda de estrategias didácticas que llamen la atención de todo el estudiantado es un reto con el que se enfrenta el docente día tras día, pero a su vez es una tarea que se debe realizar y que garantiza clases más atractivas y probablemente un mejor rendimiento académico. Según Tonconi (2010), la motivación es la fuerza interior que dinamiza y energiza al individuo en dirección de una meta y unos resultados específicos. Extendiendo esta afirmación al campo académico y laboral en particular, asegura que tal motivación puede observarse en el comportamiento general y en el desempeño en particular, entendidos como un efecto o condición consecuente de aquella.

Asimismo, al analizar las respuestas sobre el interés de los jóvenes y las jóvenes en utilizar la tecnología, se evidencia que la utilización de las redes sociales en los educandos es muy frecuente. La mayoría utilizan Facebook y, en menos cantidad, Twitter. Solamente tres personas no utilizan ninguna red social. Con estos resultados se demuestra que 
las redes sociales son de fácil acceso para la mayoría de estudiantes, ya sea que hagan uso de estas desde sus casas o en el mismo centro educativo. Según Mestres (2011), las redes sociales participan en la concepción de un aula más abierta, del aula como espacio flexible, real e interactivo para el aprendizaje y sus aplicaciones futuras más allá del centro educativo. La utilización de las redes en clase puede suponer un acercamiento entre docente y estudiante, puesto que puede interpretar que en el centro educativo se utiliza su "mismo lenguaje".

Todos los recursos didácticos que se utilicen en una clase van de la mano con la forma en la que el docente desarrolle sus lecciones. Para crear en el estudiantado un aprendizaje realmente significativo con el uso de las tecnologías, estas pueden ser utilizadas en la elaboración de tareas, videos, informaciones, entre otros, donde tanto profesorado como educandos se relacionen de una forma más placentera en un mismo espacio educativo.

Según Miranda (2003), los centros educativos deben asumir el compromiso de brindar acceso a la tecnología de la información -en particular a computadores y acceso al Internet- a sus estudiantes, pero complementarlo con orientación, motivación, capacitación y supervisión, para promover el desarrollo de habilidades relacionadas con los objetivos esperados.

Se conoce que no toda la información que se encuentra en Internet es buena, por ende, el docente tiene la responsabilidad de no solamente ser un mediador del conocimiento, sino también velar por que los sitios a los cuales acceden sus estudiantes durante el desarrollo de la clase sean adecuados, de esta forma obtiene un control preventivo, con el fin de hacer un buen uso de dicha fuente tecnológica. Además, es necesario desarrollar, en los grupos de estudiantes, las habilidades cognitivas y las competencias necesarias para un buen uso de las herramientas tecnológicas.

En la actualidad, la sociedad está siendo impactada grandemente por las tecnologías de la información, por lo que se hace imprescindible que los procesos educativos respondan a las transformaciones que, en la vida cotidiana y en el mundo del trabajo, se producen de forma constante. Por ello, el personal docente debe tener claridad sobre las directrices que orientan la práctica profesional, de tal forma que se acople a las necesidades de la diversidad y de la complejidad, porque el contexto sociocultural en el cual está inmersa la persona es, sin duda, un contexto tecnológico que le delimita, en buena medida, sus formas de aprender.

\section{Conclusiones}

Por medio de los resultados obtenidos y su respectivo análisis, se pueden rescatar ciertos aspectos relevantes en cuanto a la utilización de los recursos tecnológicos para el desarrollo de las lecciones de Ciencias de la muestra que se estudió: 
Se identificó que el personal docente de otras instituciones implementa algunos recursos tecnológicos, para estudiar los temas uso racional de la energía, la atmósfera, fenómenos del Niño y de la Niña, la capa de ozono, hidrósfera y deterioro del planeta tierra; sin embargo, no establecen la relación con el eje transversal cultura ambiental para el desarrollo sostenible, aunque tenga relación con la temática, es decir, no se implementan actividades de manera que los educandos se apropien de la realidad, en la detección y solución de problemas, en el ámbito local, pero con visión planetaria, tal como lo solicita el MEP.

- También se concluye que la falta de familiaridad y conocimiento, por parte de la docente, respecto al uso de diferentes recursos tecnológicos para apoyar el proceso educativo, influye directamente en el uso de estos en las aulas, haciendo de las clases un ambiente menos atractivo y motivador para el logro de los objetivos planteados.

- Al identificar las preferencias y el interés del grupo de estudiantes de la sección observada en cuanto a la utilización de recursos tecnológicos, se evidenció que no solamente está involucrado en el uso de estas TIC, sino también que les gustaría aprender con estas estrategias, a fin de hacer la clase más amena y familiarizada con el medio en el que se desenvuelven fuera del aula.

- El hecho de que la mayoría de docentes de otras instituciones y los estudiantes encuestados tengan una actitud positiva hacia el uso de las TIC se convierte en una fortaleza, que permite potenciar actividades donde se utilice la tecnología para trabajar diferentes temas y también para vivenciar el eje transversal cultura ambiental para el desarrollo sostenible, ya que además de ser un deber de cada docente implementarlo, es importante hacer conciencia en el estudiantado acerca de estos temas relacionados directamente con su realidad y que en la actualidad son relevantes por la situación que atraviesa el planeta.

- Se puede afirmar que el uso de la tecnología es un tema que trae controversia a la hora de decidir su implementación en la educación, como se evidenció en los resultados obtenidos en la investigación. Por un lado, en las opiniones estudiantiles se muestra gran interés por recibir las lecciones utilizando recursos tecnológicos, debido a que en su entorno directo se relacionan constantemente con estos y son de gran atractivo para ellos; pero, por otra lado, se tiene la opinión docente, que no muestra interés alguno por el tema, además de ver en el uso de tecnología educativa muchas limitaciones.

\section{Recomendaciones}

\section{A las instituciones educativas:}

- Incentivar el uso de las TIC ya que las nuevas generaciones nacen, crecen y son educadas en un contexto tecnológico, donde los procesos de enseñanza y aprendizaje, asistidos por medios tecnológicos, tienen una dimensión de interacción cultural sin precedentes, porque no existen fronteras ni límites. 
- Vivenciar la educación ambiental como parte esencial del currículo educativo, de manera que no sea concebida como una disciplina que segregue el conocimiento sino, como la plantea el Ministerio de Educación Pública, eje transversal que permita la inclusión de los contenidos disciplinares junto con aspectos relacionados con el ambiente, desarrollando así, valores, actitudes, aptitudes en pro del mejoramiento de la calidad de vida humana y del ambiente.

\section{A los docentes:}

- Tener claridad de que las TIC son una valiosa herramienta para enriquecer el aprendizaje, pero siempre que se utilicen metodologías participativas y constructivistas.

- Inculcar en sus estudiantes valores que les motiven a apreciar los recursos naturales, de manera que se generen actitudes críticas, responsables y comprometidas con el ambiente en estas personas en formación, tomando en cuenta un desarrollo en el cual se promueve el cuidado del ambiente.

\section{Referencias}

Bautista, J. (2007). Importancia de las TIC en el proceso de enseñanza aprendizaje [Mensaje en un blog]. Recuperado de http://comunidadesvirtuales.obolog.com/importancia-ticproceso-ensenanza-aprendizaje-40185

Boff, L. (19 de marzo, 2007). Calentamiento global y la existencia de una nueva moralidad (Conferencia inaugural I ciclo lectivo) llevada a cabo en la Universidad Nacional, Heredia, Costa Rica.

Borderías, M. y Martín, E. (2006). Medio ambiente urbano. Madrid: UNED.

Chadwick, C. (2001). La psicología de aprendizaje del enfoque constructivista. Revista Latinoamericana de Estudios Educativos, 31(4),111-126. Recuperado de http://redalyc. uaemex.mx/src/inicio/ArtPdfRed.jsp?iCve=27031405

Delgado, M. y Solano, A. (2009). Estrategias didácticas creativas en entornos Virtuales para el aprendizaje. Actualidades Investigativas en Educación, 9(2),1-21. Recuperado de http:// revista.inie.ucr.ac.cr/uploads/tx magazine/virtuales.pdf

Díaz, F. y Hernández, G. (2002). Estrategias docentes para un aprendizaje significativo. Una interpretación constructivista. ( $2^{\mathrm{a}}$ ed.). México: Editorial Mc-Graw Hill.

Galvis, A. H. (junio, 2004). Oportunidades educativas de las TIC. Metacursos soluciones eLearning innovadoras. Recuperado de http://www.colombiaaprende.edu.co/html/ investigadores/1609/articles-73523 archivo.pdf 
URL: http://www.una.ac.cr/educare

González, A. (2005). Las tecnologías de la información y la educación. Observatorio para la CiberSociedad [Documento en un blog]. Recuperado de http://www.cibersociedad.net/ archivo/articulo.php?art=201

Machado, S. (2002). La importancia de la educación ambiental en la protección de la biodiversidad de Brasil. Recuperado de http://www.docstoc.com/docs/22268064/La-importancia-de-laeducación-ambiental-en-la-protección

Martínez, R. (2010, enero-junio). La importancia de la educación ambiental ante la problemática actual. Revista electrónica Educare, 14(1), 97-111. Recuperado de http://www.una.ac.cr/ educare/PDF/PDF-VOL-XIV-N1-2010/11-MARTINEZ-LA-IMPORTANCIA-VIGENTE.pdf

Mestres, L. (2011). Redes sociales y educación: hacia la innovación didáctica. Educaweb. com. Recuperado de http://www.educaweb.com/noticia/2011/01/31/redes-socialeseducacion-innovacion-didactica-14583.html

Ministerio de Educación Pública. (2005). Programa de estudio ciencias III ciclo. San José, Costa Rica: Autor

Miranda, C. (2003). Riesgos y aspectos negativos de TIC en educación. Educar.org. Recuperado de http://portal.educar.org/foros/riesgos-y-aspectos-negativos-de-tic-en-educacion

Olier, K. (s. f.). Efectos de un programa de educación virtual sobre los conocimientos de los docentes acerca el uso de las nuevas tecnologías de la información y comunicación con fines educativos. (Tesis de Licenciatura). Barranquilla: Universidad del Norte. Recuperado de http://www. udenar.edu.co/viceacademica/EDUCACION EN ESPACIOS VIRTUALES/EFECTOS DE UN PROGRAMA DE EDUCACION VIRTUAL.doc

Palomo, R., Ruiz, J. y Sánchez J. (2006). Las TIC como agentes de innovación educativa. España: Dirección General de Innovación Educativa y Formación del Profesorado.

Romero, G.-A. (marzo, 2009). La utilización de las nuevas tecnologías como recurso educativo en el aula. Innovación y experiencias educativas, 16, 1-9. Recuperado de http://www.csi-csif.es/ andalucia/modules/mod_ense/revista/pdf/Numero_16/GUSTAVO ADOLFO_ROMERO_2. pdf

Pérez, G. (2007). Desafíos de la investigación cualitativa. Recuperado de http://www.rmm.cl/ usuarios/pponce/doc/200711151514230.6conferencia gloria perez serrano.pdf

Salgado, C. (2009). Políticas, estrategias y planes regionales, subregionales y nacionales para el desarrollo sostenible y la educación ambiental en América Latina y el Caribe. Decenio de las Naciones Unidas de la Educación para el desarrollo sostenible 2005-2014. Santiago: OREAL/ UNESCO. Recuperado de http://unesdoc.unesco.org/images/0018/001819/181906s.pdf 
Tonconi, J. (enero, 2010). Factores que influyen en el rendimiento académico y la deserción de los estudiantes de la Facultad de Ingeniería Económica de la Una-Puno, periodo 2009. Cuadernos de Educación y Desarrollo, 2(11). Recuperado de http://www.eumed.net/rev/ ced/11/jtq.htm

\section{Cómo citar este artículo, según APA:}

Matarrita, L., Serrano, J., Torres, M. I. y León, G. (2012). Factibilidad de la utilización de recursos tecnológicos en la implementación del eje transversal cultura ambiental para el desarrollo sostenible en la Educación General Básica de Costa Rica. Revista Electrónica Educare, 16(3), 127-143. Consultado de http://www.revistas.una.ac.cr/index.php/EDUCARE/issue/current

Nota: Para citar este artículo en otros sistemas puede consultar el hipervínculo "Como citar el artículo" en la barra derecha de nuestro sitio web:

http://www.revistas.una.ac.cr/index.php/EDUCARE/index 